\title{
Introducing Polyautoimmunity: Secondary Autoimmune Diseases No Longer Exist
}

\author{
Adriana Rojas-Villarraga, Jenny Amaya-Amaya, Alberto Rodriguez-Rodriguez, \\ Rubén D. Mantilla, and Juan-Manuel Anaya
}

Center for Autoimmune Diseases Research (CREA), School of Medicine and Health Sciences, Universidad del Rosario, Kr 24 \# 63 C 69 Third Floor, Bogotá, Colombia

Correspondence should be addressed to Adriana Rojas-Villarraga, samanda.rojas@urosario.edu.co

Received 13 October 2011; Accepted 30 November 2011

Academic Editor: Mario García-Carrasco

Copyright (C 2012 Adriana Rojas-Villarraga et al. This is an open access article distributed under the Creative Commons Attribution License, which permits unrestricted use, distribution, and reproduction in any medium, provided the original work is properly cited.

\begin{abstract}
Similar pathophysiological mechanisms within autoimmune diseases have stimulated searches for common genetic roots. Polyautoimmunity is defined as the presence of more than one autoimmune disease in a single patient. When three or more autoimmune diseases coexist, this condition is called multiple autoimmune syndrome (MAS). We analyzed the presence of polyautoimmunity in 1,083 patients belonging to four autoimmune disease cohorts. Polyautoimmunity was observed in 373 patients (34.4\%). Autoimmune thyroid disease (AITD) and Sjögren's syndrome (SS) were the most frequent diseases encountered. Factors significantly associated with polyautoimmunity were female gender and familial autoimmunity. Through a systematic literature review, an updated search was done for all MAS cases (January 2006-September 2011). There were 142 articles retrieved corresponding to 226 cases. Next, we performed a clustering analysis in which AITD followed by systemic lupus erythematosus and SS were the most hierarchical diseases encountered. Our results indicate that coexistence of autoimmune diseases is not uncommon and follows a grouping pattern. Polyautoimmunity is the term proposed for this association of disorders, which encompasses the concept of a common origin for these diseases.
\end{abstract}

\section{Introduction}

Autoimmune diseases (ADs) have particular clinical characteristics and phenotypes depending on their nature (i.e., organ specific or systemic diseases). However, there is strong evidence that ADs share several clinical signs and symptoms, physiopathological mechanisms, and environmental and genetic factors, and this fact indicates that they have a common origin [1], which has been called the autoimmune tautology.

The clinical evidence of the autoimmune tautology highlights the cooccurrence of distinct ADs within an individual (i.e., polyautoimmunity) [1]. In an earlier paper, we described the foremost systematic literature review grouping all published cases of multiple autoimmune syndromes (MAS), defined by the presence of three or more welldefined ADs in a single patient, up until 2006. Initially, MAS was first mentioned by Pirofsky and Vaughn [2] and deeply described by Humbert and Dupond [3]. They provided a taxonomy for the cooccurrent phenotypes $[4,5]$. MAS together with polyglandular autoimmune syndromes (PAS) II through IV, which are all MAS, represent the best example of polyautoimmunity [4]. Three basic, large clusters were found. Each of them had a predominant disease that was named the "chaperones" of autoimmunity, namely, autoimmune thyroid disease (AITD), Sjögren's syndrome (SS), and systemic lupus erythematosus (SLE). Study of the literature and clinical observation led to a similar clustering nomenclature which included the thyrogastric cluster and lupus-associated cluster $[6,7]$.

This coexistence of ADs in a single individual has lead researchers to consider different terms like autoimmune diathesis [8] or kaleidoscope of autoimmunity [9] both of which point to a common genetic background of ADs [10, 11]. The genetic basis of autoimmune clustering can depict part of the patterns of clustering across the spectrum of the implicated diseases [6]. 
Polyautoimmunity is also important for the current discussion because it may influence on the severity of ADs. In fact, some authors argue that there is a more severe presentation of a particular $\mathrm{AD}$ when polyautoimmunity is present [12-14], while others have found no influence or even a better prognosis in such cases [15-17].

In order to demonstrate one of the edges of autoimmune tautology, this study describes the presence of polyautoimmunity in four cohorts of ADs and analyses the main factors associated with its presence. In addition, an update of MAS cases is presented.

\section{Material and Methods}

2.1. Study Subjects. Four previously published series of ADs patients were evaluated. All of them had cross-sectional designs analyzing the presence of polyautoimmunity in patients with SLE [18], rheumatoid arthritis (RA) [19], multiple sclerosis (MS) [20], and systemic sclerosis (SSc) [21]. All the patients were recruited from a multicenter cohort of ADs patients followed at the Center for Autoimmune Diseases Research (CREA) at the Universidad del Rosario in Bogota, Colombia. Patients fulfilled the American College of Rheumatology (ACR) criteria for the classification of SLE, SSc, and RA [22-24] and McDonald's criteria for MS [25]. The institutional review board approved the study design.

Each patient was evaluated by a rheumatologist or a neurologist depending on the case. The information on patient demographics and cumulative clinical and laboratory data was obtained by physical examination, interview, and chart review. All data were collected in an electronic and secure database.

There were $23 \mathrm{ADs}$ investigated in the cohorts based on international validated criteria including autoimmune adrenal insufficiency (AAI: Addison's disease), alopecia areata (AA), autoimmune hepatitis (AIH), AITD, antiphospholipid syndrome (APS), biliary inflamatory disease including primary sclerosing cholangitis and primary biliary cirrhosis (BID), celiac disease (CD), demyelinating autoimmune diseases (DAD) including transverse myelitis (TM) and MS, dermatomyositis, polymyositis (DM/PM), inflamatory bowel disease including ulcerative colitis and Crohn disease (IBD), myastenia gravis (MG), pernicious anemia (PA), pemphigus (PF), psoriasis (Pso), RA, relapsing polychondritis (RePo), sarcoidosis (Sar), SS, SSc, SLE, type 1 diabetes mellitus (T1D), vasculitis (Vas), and vitiligo (VIT).

The presence of familial autoimmunity, including the presence of the same group of ADs evaluated in the search for polyautoimmunity, was estimated by interviewing the patients and, in most of the cases, by clinical evaluation of the affected family members as previously reported [26]. Firstdegree relatives (FDR) were defined as parents and siblings.

2.2. Systematic Literature Review. An updated systematic literature review was done for all MAS cases reported from January 2006 to September 2011 (Figure 1). Publications were identified through a systematic search done by two independent experts in Pubmed. The only limits applied were Human. The [Majr] terms "multiple autoimmune diseases," "multiple autoimmune syndrome," "multiple autoimmune disease," “polyautoimunity," “co-occurrent," “cooccurrence," "coexistence," "overlap," “associated," “concurrent"; [Mesh] terms: diabetes mellitus, type 1; antiphospholipid syndrome; lupus erythematosus, systemic; arthritis, rheumatoid; arthritis, juvenile rheumatoid; arthritis, psoriatic; spondylitis, ankylosing; spondylarthropathies; Sjögren's syndrome; Churg-Strauss syndrome; giant cell arteritis; microscopic polyangiitis; cryoglobulinemia; polyarteritis nodosa; Wegener granulomatosis; scleroderma, localized; scleroderma, systemic; scleroderma, diffuse; scleroderma, limited; dermatomyositis; colitis, ulcerative; Crohn disease; inflammatory bowel diseases; anemia, pernicious; thyroiditis, autoimmune; Hashimoto disease; Graves disease; celiac disease; hepatitis, autoimmune; liver cirrhosis, biliary; cholangitis, sclerosing; myasthenia gravis; multiple sclerosis; myelitis, transverse; polychondritis, relapsing; Addison's disease; purpura, thrombocytopenic, idiopathic; psoriasis; sarcoidosis; autoimmune gastritis; alopecia areata; autoimmune pancreatitis; pemphigus vulgaris; pemphigus bulloso; pemphigus foliaceous; vitiligo; autoimmune anemia.

Finally, the systematic literature review up to 2006 was checked and updated. The previous and new cases were compiled into a new table (see Supplementary Material available online at doi:10.1155/2012/254319).

2.3. Statistical Analysis. The prevalence of coexisting ADs was figured out separately by individual. The difference in the proportion of the associated ADs between two index conditions was calculated by chi-square, multinomial test corrected by Yate's continuity depending on the case. The degrees of freedom and Cramer's V were calculated.

A multivariate analysis was done for each of the series (SLE, MS, SSc, and RA) to identify factors associated with polyautoimmunity using logistic regressions models adjusted for age, gender, and duration of disease. Adjusted odds ratios (AORs) were calculated with 95\% confidence intervals (CIs). A $P$ value of less than 0.05 was considered significant. The Hosmer and Lemeshow Goodness-of-Fit Test was applied [27].

A hierarchical cluster procedure analysis was done to identify relatively homogeneous subgroups of variables based on selected cases with MAS reported in the systematic review of the literature. The reported cases from the previous systematic review [4] were computed with the results of the updated search. The objective of this analysis was to find out which ADs agglomerate more frequently. The cluster method implemented [28] was Single Linkage Sneath and the measure of similarity was Matching Coefficient. SPSS (V17 for Windows, Chicago, IL) software was used for all the analysis.

\section{Results}

3.1. Polyautoimmunity Patients. The 1,083 individuals studied included 335 SLE, 304 RA, 154 MS, and 290 SSc patients. There were 373 patients with polyautoimmunity $(34.4 \%)$. 


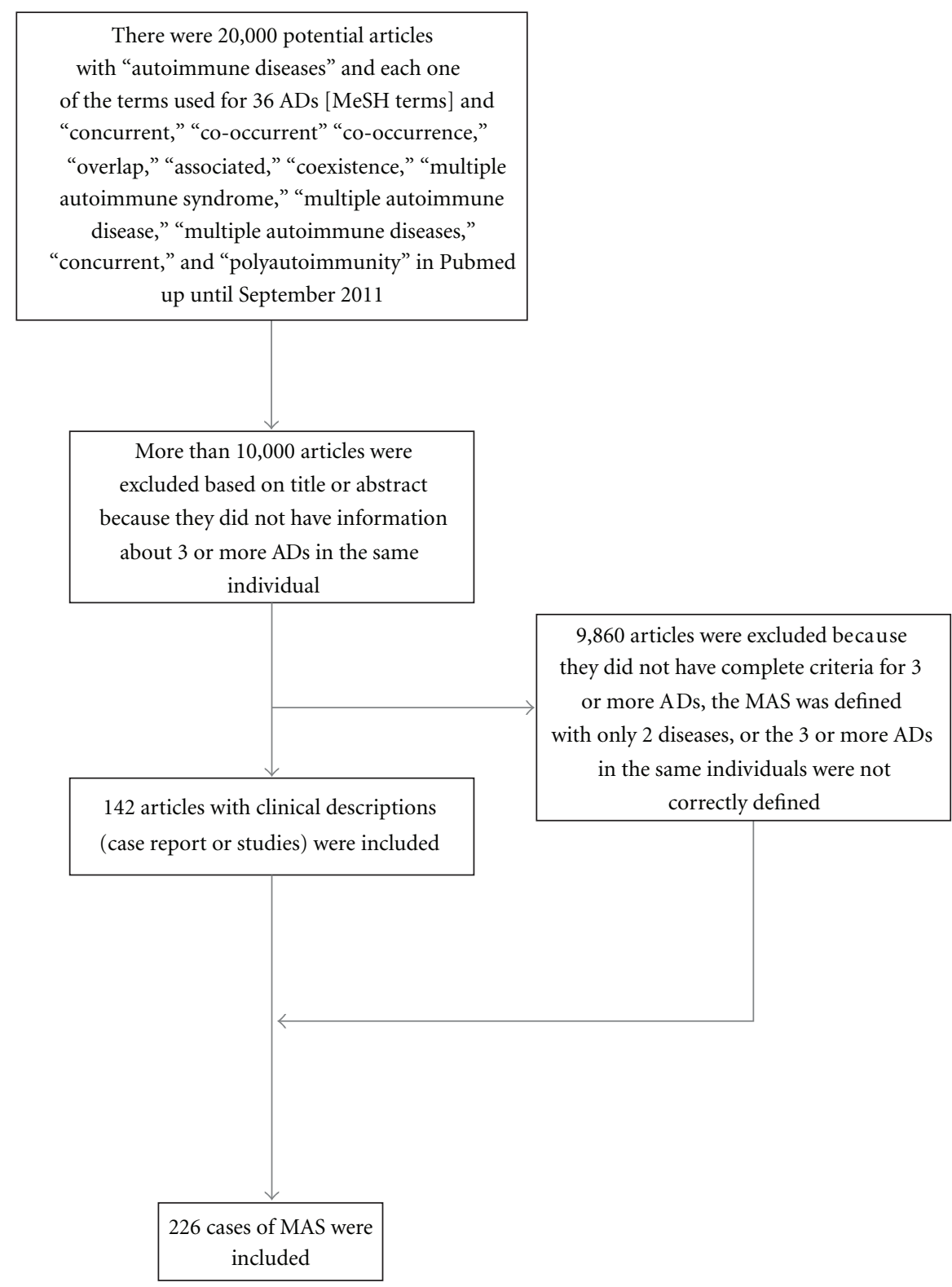

FIGURE 1: Flow chart of the systematic literature review. ADs: autoimmune diseases; MAS: multiple autoimmune syndrome.

The prevalence of polyautoimmunity was significantly different among the four $\mathrm{ADs}$, being less frequent in MS (Table 1).

AITD was the most frequent coexisting $\mathrm{AD}$ and was associated with SSc in $23 \%(N=67)$ of the cases, RA in $21 \%(N=64)$, SLE in $17.9 \%(N=60)$, and MS in $9.1 \%(N=14)$. This was followed by SS which was associated with SSc in $14.8 \%(N=43)$ of the cases, SLE in $14 \%(N=47)$, RA in $11.8 \%(N=36)$, and $\mathrm{MS}$ in $2.6 \%(N=4)$. MAS was found in $11.6 \%(N=39)$, 9.7\% $(N=28), 5.3 \%(N=16)$, and $1.9 \%(N=3)$ of SLE, SSc, RA, and MS patients, respectively.

Factors significantly associated with polyautoimmunity are depicted in Table 2. Female gender was a shared factor that was significantly associated with polyautoimmunity in the four ADs. Familial autoimmunity was significantly associated with polyautoimmunity in SLE and SSc patients.

3.2. Systematic Literature Review. The flow chart for the systematic literature review and the articles included are shown in Figure 1. A total of 142 articles corresponding to 226 cases of MAS were included.

According to the dendogram (Figure 2), the most hierarchical $\mathrm{AD}$ in the MAS cases is represented by AITD followed by SLE and SS. Otherwise, the least representative diseases in the same context are juvenile chronic arthritis (JCA), ankylosing spondylitis (AS), and RePo. Although there were several articles about combined AIH and BID polyautoimmunity, the two were not close to each other 
TABLE 1: Polyautoimmunity in 1,083 patients with four index autoimmune diseases.

\begin{tabular}{|c|c|c|c|c|c|c|c|c|c|c|c|c|}
\hline & \multicolumn{2}{|c|}{ SLE } & \multicolumn{2}{|c|}{ RA } & \multicolumn{2}{|c|}{ MS } & \multicolumn{2}{|c|}{ SSc } & \multirow{2}{*}{ chi } & \multirow{2}{*}{$\mathrm{df}$} & \multirow{2}{*}{$P$} & \multirow{2}{*}{ Cramer's V } \\
\hline & $N$ & $\%$ & $N$ & $\%$ & $N$ & $\%$ & $N$ & $\%$ & & & & \\
\hline $\mathrm{N}$ & 335 & 30.9 & 304 & 28.1 & 154 & 14.2 & 290 & 26.8 & & & & \\
\hline Polyautoimmunity & 136 & 40.6 & 98 & 32.2 & 21 & 13.6 & 118 & 40.7 & 40.81 & 3 & 0.001 & 0.19 \\
\hline AITD & 60 & 17.9 & 64 & 21.1 & 14 & 9.1 & 67 & 23.1 & 14.63 & 3 & 0.0022 & 0.11 \\
\hline SS & 47 & 14.0 & 36 & 11.8 & 4 & 2.6 & 43 & 14.8 & 16.4 & 3 & 0.0009 & 0.12 \\
\hline VIT & & & & & 2 & 1.3 & & & & & & \\
\hline APS & 48 & 14.3 & 8 & 2.6 & & & & & 25.83 & 1 & $0.001^{*}$ & 0.2 \\
\hline Primary biliary cirrosis & & & & & & & 15 & 5.2 & - & - & - & - \\
\hline MAS & 39 & 11.6 & 16 & 5.3 & 3 & 1.9 & 28 & 9.7 & 17.99 & 3 & 0.0004 & 0.12 \\
\hline
\end{tabular}

SLE: systemic lupus erythematosus; RA: rheumatoid arthritis; MS: multiple sclerosis; SSc: systemic sclerosis; AITD: autoimmune thyroid disease; SS: Sjögren's syndrome; VIT: vitiligo; APS: antiphospholipid syndrome; MAS: multiple autoimmune syndrome; chi: chi-square test; df: degree freedom; $P: P$ value; ${ }^{*}$ Yates chi-square.

TABLE 2: Significant factors associated with polyautoimmunity.

\begin{tabular}{|c|c|c|c|c|c|c|c|c|}
\hline & \multicolumn{2}{|l|}{ SLE } & \multicolumn{2}{|l|}{ RA } & \multicolumn{2}{|l|}{ MS } & \multicolumn{2}{|l|}{ SSc } \\
\hline & AOR; CI95\% & $P$ & AOR; CI95\% & $P$ & AOR; CI95\% & $P$ & AOR; CI95\% & $P$ \\
\hline Female gender & $2.3 ; 1.03-5.15$ & 0.043 & $1.8 ; 1.22-6.31$ & 0.015 & $8.5 ; 1.02-70.8$ & 0.048 & $9.08 ; 2.09-39.3$ & 0.003 \\
\hline Familial autoimmunity & $1.61 ; 1.14-2.28$ & 0.007 & NS & & NS & & $2.62 ; 1.24-5.54$ & 0.01 \\
\hline Articular Involvement & $2.02 ; 1.26-3.23$ & 0.003 & NS & & $\mathrm{NE}$ & & NS & \\
\hline Anti-Ro positivity & $1.54 ; 1.10-2.16$ & 0.013 & NS & & $\mathrm{NE}$ & & NS & \\
\hline Cardiovascular disease & NS & & $2.2 ; 1.17-3.94$ & 0.014 & $\mathrm{NE}$ & & NE & \\
\hline ANAs & NS & & $2.0 ; 1.08-3.84$ & 0.027 & NS & & & \\
\hline SSEP & $\mathrm{NE}$ & & $\mathrm{NE}$ & & $10.86 ; 1.31-89.6$ & 0.027 & NE & \\
\hline
\end{tabular}

SLE: systemic lupus erythematosus; RA: rheumatoid arthritis; MS: multiple sclerosis; SSc: systemic sclerosis; AOR: adjusted odd ratio; CI95\%: confidence interval; ANAs: antinuclear antibodies; SSEP: somatosensory evoked potentials; NS: nonsignificant; NE: not evaluated.

on the dendogram nor did they show a suitable degree of agreement.

\section{Discussion}

Herein, we report one of the largest series of polyautoimmunity with an emphasis on its associated factors. Some authors had shown that so far the evidence suggesting that ADs tend to coexist within both individuals and families was anecdotal corresponding to the concept of autoimmune diathesis [29]. By grouping diverse ADs in the same patient (i.e., polyautoimmunity) including organ specific (i.e., MS) and systemic ADs, we have demonstrated that they are true associations as a part of the autoimmune tautology rather than the chance findings that were previous hypothesized [4].

Polyautoimmunity is a term that can group all the taxonomy terms referring to coexistence of well-defined ADs in a single individual because some of the terms previously used are confusing and exclude various associations. Polyautoimmunity was used by Sheenan and Stanton-King [30] for the first time while describing a patient with ITP, PA, AITD, SSc, pancreatic exocrine insufficiency, and CD before dying from vasculitic complications. The case they depicted corresponds to a typical MAS, which is already included in the term polyautoimmunity. Also, when patients fully develop two or more diseases simultaneously or sequentially, these diseases have frequently been classified as overlap syndromes; some of these were frequent enough to have been given names like rhupus and sclerodermatomyositis [31]. In another case, some authors have historically postulated that mixed connective tissue disease (MCTD) is a very homogeneous entity with shared clinical manifestations rather than shared diseases or autoantibodies [32, 33], while others have not. The existence of MCTD as a distinct disease entity has been a matter of controversy among researchers since it was first described $[34,35]$. In fact, the coexistence of several sets of classification criteria for MCTD indicates how difficult it is to give a precise definition of the disease [33]. In addition, some patients will develop SLE, SSc, or RA, during the course of MCTD, and some will present with a longstanding MCTD [36]. In real-life conditions, searching for the specific phenotypes (antibodies and clinical) over the course of disease and constantly looking for associated ADs, including organ specific and systemic, are more useful for developing an exact description of polyautoimmunity than taxonomic discussions.

The fact that many ADs share a similar underlying pathology and have a tendency to cluster supports the involvement of shared susceptibility genes and similar molecular mechanisms. In fact, recent studies have identified several common genes associated with multiple ADs supporting the presence of autoimmunity genes as part of the autoimmune tautology [10, 37, 38]. 
Rescaled distance cluster combine

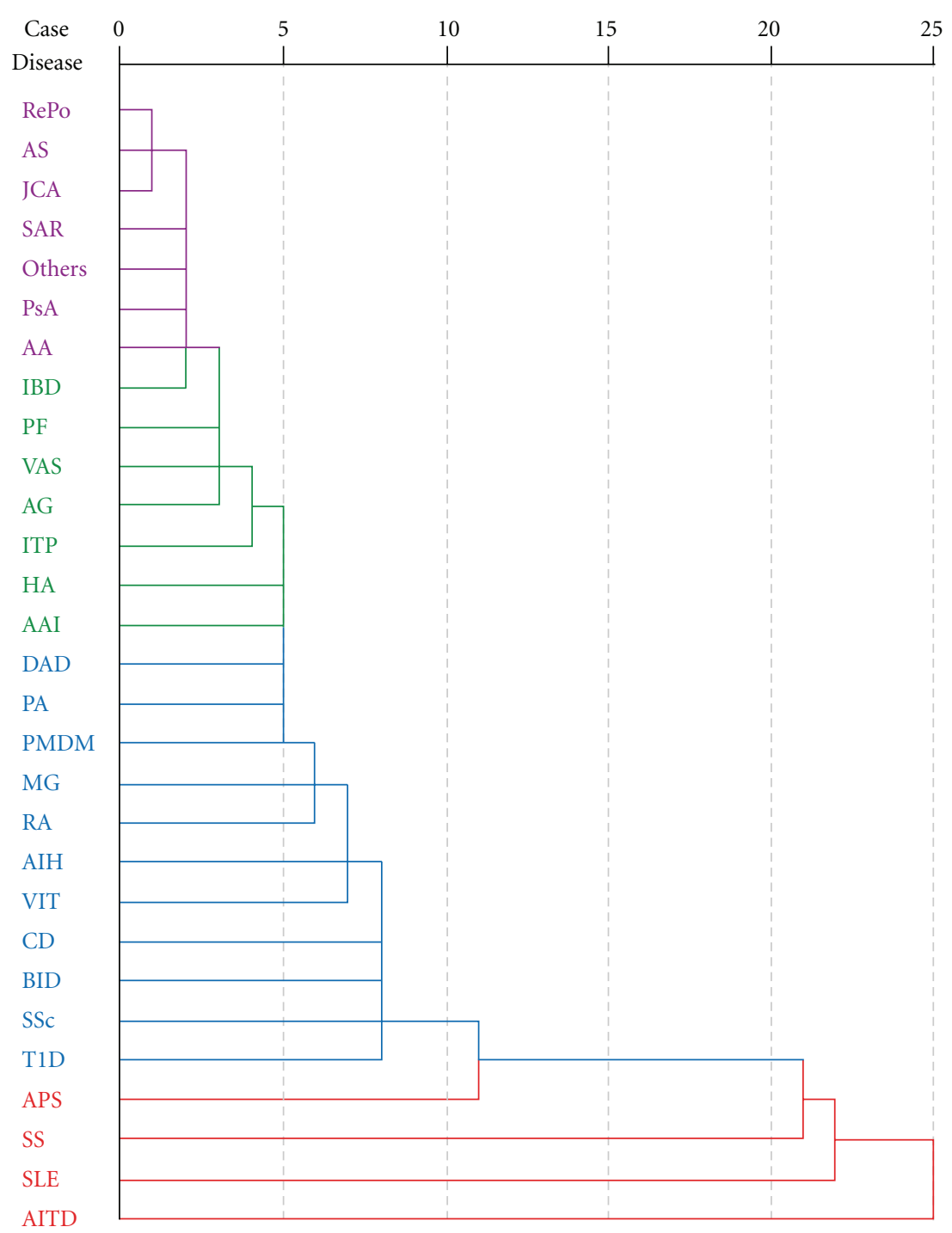

Figure 2: Cluster analysis dendogram. Each node represets a stage from the clustering process. There were four clusters. The most hierarchical was composed of four ADs. AITD: autoimmune thyroid disease (including thyroiditis, Hashimoto disease, Graves disease); SLE: systemic lupus erythematosus; SS: Sjögren's syndrome; APS: antiphospholipid syndrome; T1D: type 1 diabetes mellitus; SSc: scleroderma (including localized, systemic, diffuse, limited); BID: billiary inflammatory disease (including primary biliary cirrhosis, primary sclerosing cholangitis); CD: celiac disease; VIT: vitiligo; AIH: autoimmune hepatitis; RA: rheumatoid arthritis; MG: myasthenia gravis; PMDM: polymyositis/dermatomyositis; PA: pernicious anemia; DAD: demyelinating autoimmune diseases (including multiple sclerosis, transverse myelitis, optic neuromyelitis); AAI: autoimmune adrenal insufficiency (Addison disease); HA: autoimmune anemia; ITP: idiopathic thrombocytopenic purpura; AG: autoimmune gastritis; VAS: vasculitis (including Churg-Strauss syndrome, giant cell arteritis, microscopic polyangiitis, cryoglobulinemia, polyarteritis nodosa, Wegener granulomatosis); PF: pemphigus (including vulgaris, bulloso, foliaceous); IBD: inflammatory bowel disease (including ulcerative colitis, Crohn's disease); AA: alopecia areata; PsA: psoriasis (including psoriatic arthritis); SAR: sarcoidosis; JCA: juvenile chronic arthritis; AS: ankylosing spondylitis; RePo: relapsing polychondritis.

Familial autoimmunity and female gender were confirmed as risk factors for polyautoimmunity. Female gender was a shared factor associated with polyautoimmunity in the four index conditions here studied. This fact gives us a glimpse of one facet of the shared commonalities between ADs. The majority of ADs predominate in females [39] and constitute a leading cause of death among young and middleaged women [40]. In searching for a reason behind female predominance, most attention has focused on hormonal changes while other factors have included genetic differences, both direct (i.e., influence of genes on sex chromosomes) and indirect such as microchimerism, as well as gender differences in lifestyle $[39,41]$. Our results support previous studies including a meta-analytic approach demonstrating that most of the patients in 54 studies quantifying the coexistence of ADs among 4 selected ADs were female [29]. 
In addition, some authors have shown in specific ADs that women are distinguished from men by higher frequencies of concurrent immune diseases [42, 43].

AITD was the most frequent polyautoimmunity found in our series of 1,083 patients. This finding was supported by the analysis of the systemic literature review and depicted in the dendogram (Figure 2) where AITD was the main "chaperon" of autoimmunity. AITD has been described as the most prevalent $\mathrm{AD}$ as well as being associated with other organ-specific and non-organ-specific ADs [44].

Possible explanations for the relationship of these ADs include (a) immunomodulatory effects of antithyroid antibodies, (b) molecular mimicry between thyroid and disease-specific epitopes, and (c) a genetic link between antithyroid autoimmunity and the susceptibility to $\mathrm{AD}[45]$. In population-based database studies, other authors have demonstrated that AITD is frequently associated with other ADs [46]. All of this information indicates that AITD is clinically important in the context of autoimmunity and it is mandatory for screening patients with hypothyroidism or hyperthyroidism symptoms for the autoimmune etiology when there is suspicion of the coexistence of AITD with another $\mathrm{AD}[44]$.

The prevalence of SS was demonstrated to be high and in fact the second most frequently associated $\mathrm{AD}$ in our series as well as in the MAS cases through the literature review. Many authors have recognized that it is quite difficult to categorize concomitant SS as primary or secondary, and there is disagreement about this issue in the literature [47]. Other authors believe that salivary changes in patients with an $\mathrm{AD}$ (i.e., SLE) might reflect a multisystem presentation of the disease [48]. Regarding the association of SS with other ADs, some authors have argued that the etiopathogenic mechanism for the simultaneous or sequential development of multiple $\mathrm{ADs}$ in one individual is not well understood [49]. The concept is more developed nowadays, and the idea that common genetic backgrounds and additional immunogenetic, environmental, or hormonal factors are responsible for the formation of subsets of $\mathrm{AD}$ clusters is becoming more established.

We previously evaluated the prevalence of SS in a large series of patients with SLE $(n=969)$ and the potential risk factors for this association [50]. SS patients fulfilled the American-European classification criteria (the presence of anti-Ro antibodies or a positive minor salivary gland biopsy was mandatory). There were $9.3 \%$ patients with SS, $42 \%$ had familial autoimmunity, of which $7 \%$ had familial SS as compared to $2 \%$ in the group of SLE without SS. The factors significantly associated with SS in SLE were familial SS, anti-La and anti-Ro antibodies, as well as pulmonary involvement. Anti-Sm antibodies and Colombian origin (i.e., ethnicity) were protective factors. Our results together with other series [51,52] using similar strict classification criteria indicate that the prevalence of SS in SLE is close to $10 \%$. SLE-SS appears to constitute a subgroup of patients with distinct clinical, serologic, pathologic, and immunogenetic features, in whom SS is expressed as an associated entity and is largely similar to what has been called primary SS [52]. Clinical and immunological factors observed in our study might serve as predictors for this association. Because variations in both additive and nonadditive genetic factors and the environmental variance are specific to the investigated population, family history of autoimmunity and patient origin are important characteristics to be considered.

While the currently most accepted classification criteria [53] designate these cases as "secondary" SS, the terms "overlapping" or "associated" SS are frequently used in the literature to describe them [49]. We consider these terms to be confusing and propose that SS always be taken into account and properly investigated in patients diagnosed with any $\mathrm{AD}$ because of the high possibility of the presence of the concomitant, well-defined phenotypes as demonstrated here in the context of polyautoimmunity or as demonstrated previously with a prevalence near to $10 \%$ in SLE patients when strict classification criteria are used.

It is of interest that the primary/secondary designation for classification of APS was introduced by rheumatologists who already used the primary/secondary terms to differentiate subgroups of patients with SS. In introducing the primary APS (PAPS) subgroup, it was unclear whether one would expect that the clinical features, disease course, or management of patients would be different based on their subclassification [54]. In fact, the international (Sydney) consensus statement on an update of the classification criteria for definite APS [55] advises against using the term "secondary" APS. They did not find differences in the clinical consequences of antiphospholipid antibodies among patients in these two categories (Evidence Level I). They state most patients with the so-called secondary APS have SLE. However, they discuss that it is unknown if APS and SLE are two diseases coinciding in an individual, if underlying SLE offers a setting for the development of APS, or if APS and SLE represent two elements of the same process [55]. Some cases with "secondary" APS are classified as lupus like disease (LLD). The Sydney consensus raised up that the interface between SLE, LLD, and APS merits further consideration. Finally, the consensus states that rather than distinguishing between patients with PAPS and secondary APS, documenting the coexistence of SLE (or other disease) is more advantageous for classification and that the disorder associated with APS, such as SLE, be listed; hence, one would report "APS associated with SLE" or "APS associated with rheumatoid arthritis" rather than "secondary APS" [54]. Studies showing no significant differences between PAPS and SAPS were cited. Patients with APS plus SLE and PAPS have similar clinical profiles, although heart valve disease, hemolytic anemia, low $\mathrm{C} 4$ levels, and neutropenia seem to be more common in patients with APS plus SLE [56].

Indeed evidence that there are any differences in presentation or course of PAPS versus SAPS is not persuasive $[55,56]$, and this has led to the suggestion that PAPS/SAPS designations be replaced by APS and "APS associated with the name of the autoimmune disease." It might suggest that such a distinction exists if there are differences in clinical complications, the timing of these complications, prognosis, or frequency of positive anticardiolipin, lupus anticoagulant, or other autoantibody tests. Studies that have addressed this question have found no difference in any of 
these parameters $[57,58]$. As an instance, some authors have compared intima-media thickness (IMT), arterial stiffness, and presence of plaques in APS patients and controls. A significant difference was found between IMT, arterial stiffness, and the presence of plaques in patients and controls, but no differences in these parameters were found between patients with primary APS and those with secondary APS [59]. Additional arguments have been raised for some authors $[60,61]$ who have explored the concept of an intermediate APS (having at least one but less than four of the 11 criteria for SLE) and did not found differences between PAPS, intermediate, and the so-called secondary APS when comparing the prevalence of the thrombotic or pregnancy manifestations.

The true prevalence of the development of PAPS in SLE will require decades of followup for this determination. The distinction between PAPS and SAPS can be difficult and at times seems a rather artificial convention [60]. It may be underestimated by some studies that have a followup period that is shorter than the interval between PAPS and SLE diagnoses noted in most case reports [60]. We agree on the proposition of the Sydney committee [55] against using the term "secondary" APS and encourage clinicians to follow adequately the patients and searching for specific phenotypic characteristic to classify patients as having polyautoimmunity.

Results with respect to the severity of the disease in patients with polyautoimmunity are not unanimous. In the case of associated SS, some authors have demonstrated as we did previously [17] that there is no influence on the course of the disease. Some have found that the appearance of SS in RA patients has no relationship with RA duration or activity [62]. Others demonstrated that the subset of patients with SLE and SS has a distinct clinical and laboratory phenotype with a lower frequency of renal disease and anti-dsDNA antibodies [63]. This has not been true for other examples of polyautoimmunity when there is a severe presentation of the diseases as is the case of associated ADs in MG patients with a severe presentation [12], a severe clinical onset of T1D and increased prevalence of other ADs in children with $\mathrm{CD}$ diagnosed before T1D [13], and a severe SLE compromise when associated with vasculitis [14].

In conclusion, we suggest searching for well-defined phenotypes by looking for clusters of $\mathrm{ADs}$ in the same individual. It is our contention that the term "secondary diseases" should not longer be used because it detracts from the reality that these patients have two or more wellestablished ADs sharing the same etiopathogenesis [64]. Our results indicate that coexistence of $\mathrm{ADs}$ is not uncommon and follows a grouping pattern. Polyautoimmunity is the term proposed for this association of disorders, which encompasses the concept of a common origin for these diseases.

\section{Conflict of Interests}

The authors declare no competing financial interests.

\section{Acknowledgments}

The authors express their gratitude to the patients who participated in this study and to their colleges at the CREA for their fruitful discussions and contributions. They also thank Paola Coral-Alvarado, Antonio Iglesias-Gamarra, and Ernesto Ojeda for their contributions in the development of their cohorts.

\section{References}

[1] J. M. Anaya, "The autoimmune tautology," Arthritis Research \& Therapy, vol. 12, no. 6, p. 147, 2010.

[2] B. Pirofsky and M. Vaughn, "Addisonian pernicious anemia with positive antiglobulin tests. A multiple autoimmune disease syndrome," American Journal of Clinical Pathology, vol. 50, no. 4, pp. 459-466, 1968.

[3] P. Humbert and J. L. Dupond, "[Multiple autoimmune syndromes]," Annales de Médecine Interne, vol. 139, no. 3, pp. 159-168, 1988.

[4] A. M. Anaya, R. Corena, J. Castiblanco, A. Rojas-Villarraga, and Y. Shoenfeld, "The kaleidoscope of autoimmunity: multiple autoimmune syndromes and familial autoimmunity," Expert Review of Clinical Immunology, vol. 3, no. 4, pp. 623$635,2007$.

[5] P. Humbert and J. L. Dupond, "The multiple autoimmune syndromes (MAS)," The British Journal of Dermatology, vol. 136, no. 3, pp. 468-469, 1997.

[6] I. R. Mackay, "Clustering and commonalities among autoimmune diseases," Journal of Autoimmunity, vol. 33, no. 3-4, pp. 170-177, 2009.

[7] S. Whittingham, U. Youngchaiyud, and I. R. Mackay, "Thyrogastric autoimmune disease. Studies on the cell mediated immune system and histocompatibility antigens," Clinical and Experimental Immunology, vol. 19, no. 2, pp. 289-299, 1975.

[8] N. R. Rose, "Autoimmune diseases: tracing the shared threads," Hospital Practice, vol. 32, no. 4, pp. 147-154, 1997.

[9] M. Lorber, M. E. Gershwin, and Y. Shoenfeld, "The coexistence of systemic lupus erythematosus with other autoimmune diseases: the kaleidoscope of autoimmunity," Seminars in Arthritis and Rheumatism, vol. 24, no. 2, pp. 105-113, 1994.

[10] J. M. Anaya, L. Gómez, and J. Castiblanco, "Is there a common genetic basis for autoimmune diseases?" Clinical and Developmental Immunology, vol. 13, no. 2-4, pp. 185-195, 2006.

[11] J. Castiblanco and J. M. Anaya, "The nature and nurture of common autoimmunity," Annals of the New York Academy of Sciences, vol. 1109, pp. 1-8, 2007.

[12] P. B. Christensen, T. S. Jensen, I. Tsiropoulos et al., "Associated autoimmune diseases in myasthenia gravis. A populationbased study," Acta Neurologica Scandinavica, vol. 91, no. 3, pp. 192-195, 1995.

[13] G. Valerio, L. Maiuri, R. Troncone et al., "Severe clinical onset of diabetes and increased prevalence of other autoimmune diseases in children with coeliac disease diagnosed before diabetes mellitus," Diabetologia, vol. 45, no. 12, pp. 1719-1722, 2002.

[14] M. Ramos-Casals, N. Nardi, M. Lagrutta et al., "Vasculitis in systemic lupus erythematosus: prevalence and clinical characteristics in 670 patients," Medicine, vol. 85, no. 2, pp. 95-104, 2006. 
[15] M. Marinó, R. Ricciardi, A. Pinchera et al., "Mild clinical expression of myasthenia gravis associated with autoimmune thyroid diseases," Journal of Clinical Endocrinology and Metabolism, vol. 82, no. 2, pp. 438-443, 1997.

[16] J. Avouac, P. Airò, P. Dieude et al., "Associated autoimmune diseases in systemic sclerosis define a subset of patients with milder disease: results from 2 large cohorts of European Caucasian patients," Journal of Rheumatology, vol. 37, no. 3, pp. 608-614, 2010.

[17] A. Rojas-Villarraga, C.-E. Toro, G. Espinosa et al., "Does Sjögren syndrome or autoimmune thyroid disease influence lupus nephritis?" Lupus, no. 19, pp. S-150, 2010.

[18] A. Rojas-Villarraga, C.-E. Toro, G. Espinosa et al., "Factors influencing polyautoimmunity in systemic lupus erythematosus," Autoimmunity Reviews, vol. 9, no. 4, pp. 229-232, 2010.

[19] A. Rojas-Villarraga, R.-A. Cifuentes, D. Botello-Corzo, A. Iglesias-Gamarra, R. D. Mantilla, and J.-M. Anaya, "Polyautoimmunity and autoimmune aggregation in rheumatoid arthritis," Arthritis and Rheumatism, vol. 62, supplement 10, 1048 pages, 2010.

[20] A. Rojas-Villarraga, E. Ojeda, R. Gómez et al., "Poliautoinmunidad y agregación de enfermedades autoinmunes en pacientes con esclerosis múltiple," Acta Neurológica Colombiana, vol. 25, no. 3, p. 174, 2009.

[21] M. Hudson, A. Rojas-Villarraga, P. Coral-Alvarado et al., "Polyautoimmunity and familial autoimmunity in systemic sclerosis," Journal of Autoimmunity, vol. 31, no. 2, pp. 156-159, 2008.

[22] F. C. Arnett, S. M. Edworthy, D. A. Bloch et al., "The American rheumatism association 1987 revised criteria for the classification of rheumatoid arthritis," Arthritis and Rheumatism, vol. 31, no. 3, pp. 315-324, 1988.

[23] E. M. Tan, A. S. Cohen, J. F. Fries et al., "The 1982 revised criteria for the classification of systemic lupus erythematosus," Arthritis and Rheumatism, vol. 25, no. 11, pp. 1271-1277, 1982.

[24] Preliminary criteria for the classification of systemic sclerosis (scleroderma), "Subcommittee for scleroderma criteria of the American rheumatism association diagnostic and therapeutic criteria committee," Arthritis and Rheumatism, vol. 23, no. 5, pp. 581-590, 1980.

[25] W. I. McDonald, A. Compston, G. Edan et al., "Recommended diagnostic criteria for multiple sclerosis: guidelines from the international panel on the diagnosis of multiple sclerosis," Annals of Neurology, vol. 50, no. 1, pp. 121-127, 2001.

[26] J.-M. Anaya, G. J. Tobon, P. Vega, and J. Castiblanco, "Autoimmune disease aggregation in families with primary Sjögren's syndrome," The Journal of Rheumatology, vol. 33, no. 11, pp. 2227-2234, 2006.

[27] D.-W. Hosmer and S. Lemeshow, Applied Logistic Regression, Wiley-Interscience, New York, NY, USA, 2nd edition, 2000.

[28] B.-S. Everitt, S. Landau, M. Leese, and D. Stahl, Cluster Analysis, Wiley, Chichester, UK, 5th edition, 2011.

[29] E. C. Somers, S. L. Thomas, L. Smeeth, and A. J. Hall, "Autoimmune diseases co-occurring within individuals and within families: a systematic review," Epidemiology, vol. 17, no. 2, pp. 202-217, 2006.

[30] N. J. Sheehan and K. Stanton-King, "Polyautoimmunity in a young woman," British Journal of Rheumatology, vol. 32, no. 3, pp. 254-256, 1993.

[31] T. S. Rodríguez-Reyna and D. Alarcón-Segovia, "The different faces of shared autoimmunity," Autoimmunity Reviews, vol. 5, no. 2, pp. 86-88, 2006.
[32] D. Alarcón-Segovia, "Shared autoimmunity: a concept for which the time has come," Autoimmunity, vol. 38, no. 3, pp. 201-203, 2005.

[33] G. C. Sharp, "The origin of mixed connective tissue disease: a stimulus for autoimmune disease research," Lupus, vol. 18, no. 12, pp. 1031-1032, 2009.

[34] M. Aringer, G. Steiner, and J. S. Smolen, "Does mixed connective tissue disease exist? Yes," Rheumatic Disease Clinics of North America, vol. 31, no. 3, pp. 411-420, 2005.

[35] M. Ruiz-Pombo, A. Selva-O'Callaghan, L. Martínez-Lostao et al., "Mixed connective tissue disease: should the diagnosis be more restrictive? Comment on the article by Bodolay et al," Rheumatology, vol. 44, no. 11, pp. 1465-1467, 2005.

[36] P. J. W. Venables, "Mixed connective tissue disease," Lupus, vol. 15, no. 3, pp. 132-137, 2006.

[37] J. -M. Anaya, X. Kim-Howard, S. Prahalad et al., "Evaluation of genetic association between an ITGAM non-7 synonymous SNP (rs1143679) and multiple autoimmune diseases," Autoimmunity Reviews, vol. 11, no. 4, pp. 276-280, 2012.

[38] H. A. Deshmukh, A. K. Maiti, X. R. Kim-Howard et al., "Evaluation of 19 autoimmune disease-associated loci with rheumatoid arthritis in a Colombian population: evidence for replication and gene-gene interaction," Journal of Rheumatology, vol. 38, no. 9, pp. 1866-1870, 2011.

[39] J. E. Oliver and A. J. Silman, "Why are women predisposed to autoimmune rheumatic diseases?" Arthritis Research and Therapy, vol. 11, no. 5, p. 252, 2009.

[40] S. J. Walsh and L. M. Rau, "Autoimmune diseases: a leading cause of death among young and middle-aged women in the United States," American Journal of Public Health, vol. 90, no. 9, pp. 1463-1466, 2000.

[41] D. Fairweather and N. R. Rose, "Women and autoimmune diseases," Emerging Infectious Diseases, vol. 10, no. 11, pp. 2005-2011, 2004.

[42] A. J. Czaja and P. T. Donaldson, "Gender effects and synergisms with histocompatibility leukocyte antigens in type 1 autoimmune hepatitis," American Journal of Gastroenterology, vol. 97, no. 8, pp. 2051-2057, 2002.

[43] L. R. Ginn, J. P. Lin, P. H. Plotz et al., "Familial autoimmunity in pedigrees of idiopathic inflammatory myopathy patients suggests common genetic risk factors for many autoimmune diseases," Arthritis and Rheumatism, vol. 41, no. 3, pp. 400405, 1998.

[44] I. Lazúrová, K. Benhatchi, J. Rovenský et al., "Autoimmune thyroid disease and autoimmune rheumatic disorders: a twosided analysis," Annals of the New York Academy of Sciences, vol. 1173, pp. 211-216, 2009.

[45] M. Szyper-Kravitz, I. Marai, and Y. Shoenfeld, "Coexistence of thyroid autoimmunity with other autoimmune diseases: friend or foe? Additional aspects on the mosaic of autoimmunity," Autoimmunity, vol. 38, no. 3, pp. 247-255, 2005.

[46] E. C. Somers, S. L. Thomas, L. Smeeth, and A. J. Hall, "Are individuals with an autoimmune disease at higher risk of a second autoimmune disorder?" American Journal of Epidemiology, vol. 169, no. 6, pp. 749-755, 2009.

[47] K. H. Katsanos, V. Saougos, M. Kosmidou et al., "Sjogren's syndrome in a patient with ulcerative colitis and primary sclerosing cholangitis: case report and review of the literature," Journal of Crohn's and Colitis, vol. 3, no. 3, pp. 200-203, 2009.

[48] J. D. Fernandes, M. M.S. Nico, V. Aoki et al., "Xerostomia in Sjögren's syndrome and lupus erythematosus: a comparative histological and immunofluorescence study of minor salivary 
glands alterations," Journal of Cutaneous Pathology, vol. 37, no. 4, pp. 432-438, 2010.

[49] E. Theander and L. T. H. Jacobsson, "Relationship of Sjögren's syndrome to other connective tissue and autoimmune disorders," Rheumatic Disease Clinics of North America, vol. 34, no. 4, pp. 935-947, 2008.

[50] J.-M. Anaya, A. Rojas-Villarraga, and C.-E. Toro, "Prevalence and risk factors for Sjögren's syndrome in systemic lupus erythematosus," Annals of the Rheumatic Diseases, vol. 68, supplement 3, p. 266, 2009.

[51] H. F. Pan, D. Q. Ye, Q. Wang et al., "Clinical and laboratory profiles of systemic lupus erythematosus associated with Sjögren syndrome in China: a study of 542 patients," Clinical Rheumatology, vol. 27, no. 3, pp. 339-343, 2008.

[52] M. N. Manoussakis, C. Georgopoulou, E. Zintzaras et al., "Sjögren's syndrome associated with systemic lupus erythematosus: clinical and laboratory profiles and comparison with primary Sjögren's syndrome," Arthritis and Rheumatism, vol. 50, no. 3, pp. 882-891, 2004.

[53] C. Vitali, S. Bombardieri, R. Jonsson et al., "Classification criteria for Sjögren's syndrome: a revised version of the European criteria proposed by the American-European Consensus Group," Annals of the Rheumatic Diseases, vol. 61, no. 6, pp. 554-558, 2002.

[54] E. N. Harris, M. Phil, and S. S. Pierangeli, "Primary, secondary, and catastrophic antiphospholipid syndrome: what's in a name?" Seminars in Thrombosis and Hemostasis, vol. 34, no. 3, pp. 219-226, 2008.

[55] S. Miyakis, M. D. Lockshin, T. Atsumi et al., "International consensus statement on an update of the classification criteria for definite antiphospholipid syndrome (APS)," Journal of Thrombosis and Haemostasis, vol. 4, no. 2, pp. 295-306, 2006.

[56] J. L. Vianna, M. A. Khamashta, J. Ordi-Ros et al., "Comparison of the primary and secondary antiphospholipid syndrome: a European multicenter study of 114 patients," American Journal of Medicine, vol. 96, no. 1, pp. 3-9, 1994.

[57] E. N. Harris and S. S. Pierangeli, "Primary, secondary, catastrophic antiphospholipid syndrome: is there a difference?" Thrombosis Research, vol. 114, no. 5-6, pp. 357-361, 2004.

[58] M. Weber, G. Hayem, M. DeBandt et al., "The family history of patients with primary or secondary antiphospholipid syndrome (APS)," Lupus, vol. 9, no. 4, pp. 258-263, 2000.

[59] C. C. Belizna, V. Richard, E. Primard et al., "Early atheroma in primary and secondary antiphospholipid syndrome: an intrinsic finding," Seminars in Arthritis and Rheumatism, vol. 37, no. 6, pp. 373-380, 2008.

[60] J. M. Grossman, "Primary versus secondary antiphospholipid syndrome: is this lupus or not?" Current rheumatology reports, vol. 6, no. 6, pp. 445-450, 2004.

[61] M. Weber, G. Hayem, M. de Bandt et al., "Classification of an intermediate group of patients with antiphospholipid syndrome and lupus-like disease: primary or secondary antiphospholipid syndrome?" Journal of Rheumatology, vol. 26, no. 10, pp. 2131-2136, 1999.

[62] D. C. Antero, A. G. M. Parra, F. H. Miyazaki, M. Gehlen, and T. L. Skare, "[Síndrome de Sjögren secundária e atividade da artrite reumatoide]," Revista da Associacao Medica Brasileira, vol. 57, no. 3, pp. 319-322, 2011.

[63] A. N. Baer, J. W. Maynard, F. Shaikh, L. S. Magder, and M. Petri, "Secondary Sjögren's syndrome in systemic lupus erythematosus defines a distinct disease subset," Journal of Rheumatology, vol. 37, no. 6, pp. 1143-1149, 2010.
[64] H. M. Moutsopoulos and N. Talal, "Connective tissue diseases: one disease or many?” Lupus, vol. 3, no. 1, pp. 5-10, 1994. 


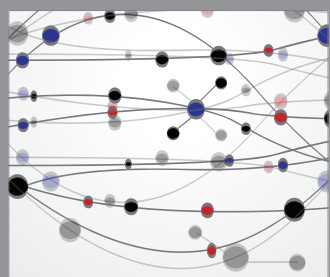

The Scientific World Journal
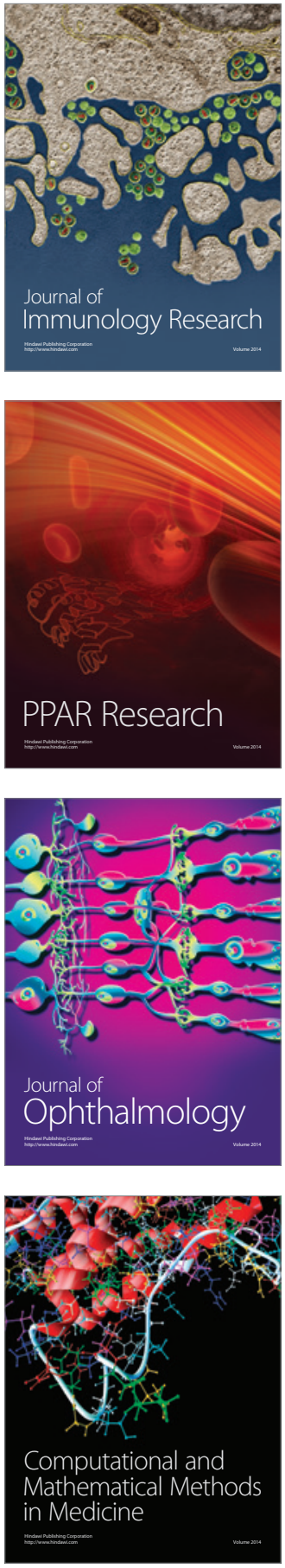

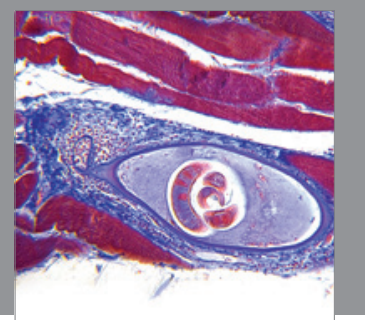

Gastroenterology

Research and Practice
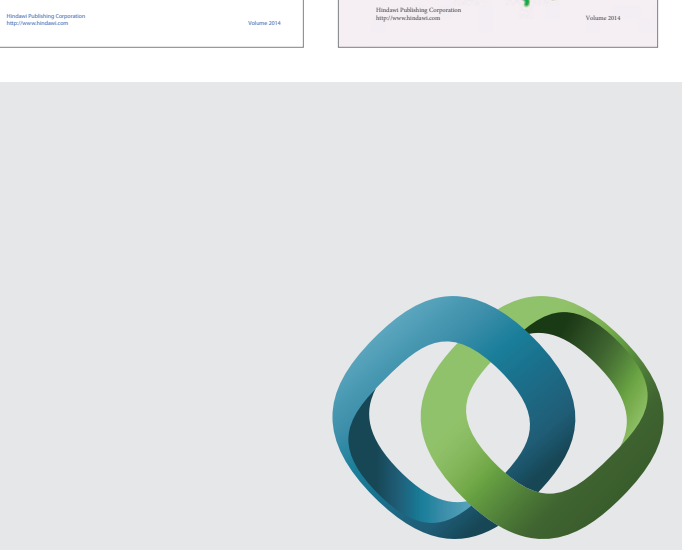

\section{Hindawi}

Submit your manuscripts at

http://www.hindawi.com
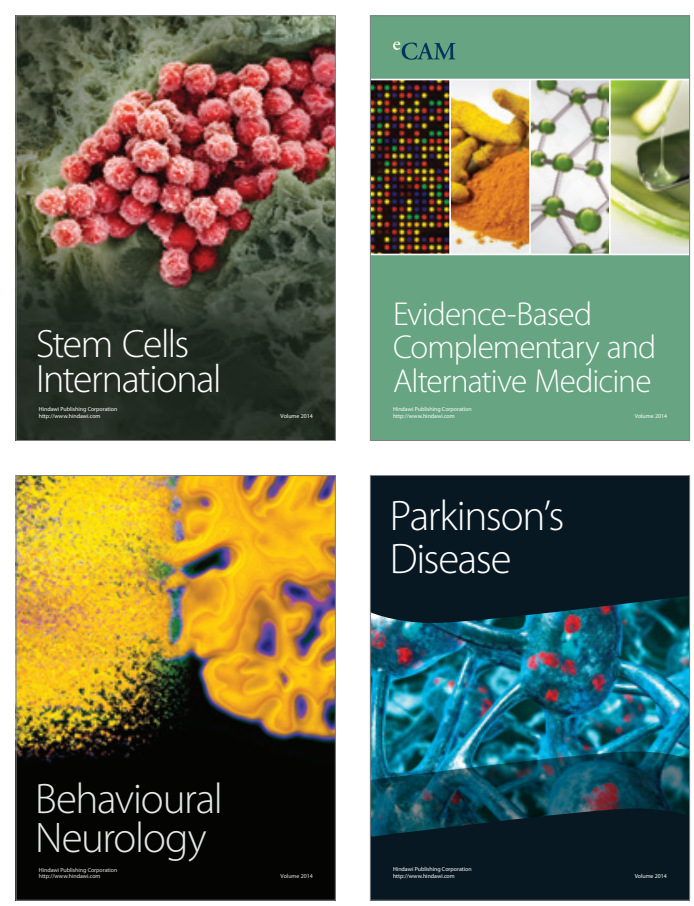

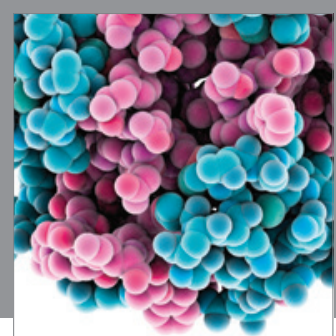

Journal of
Diabetes Research

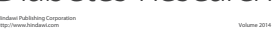

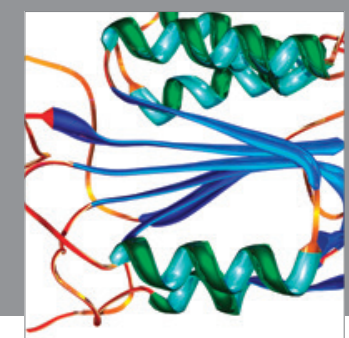

Disease Markers
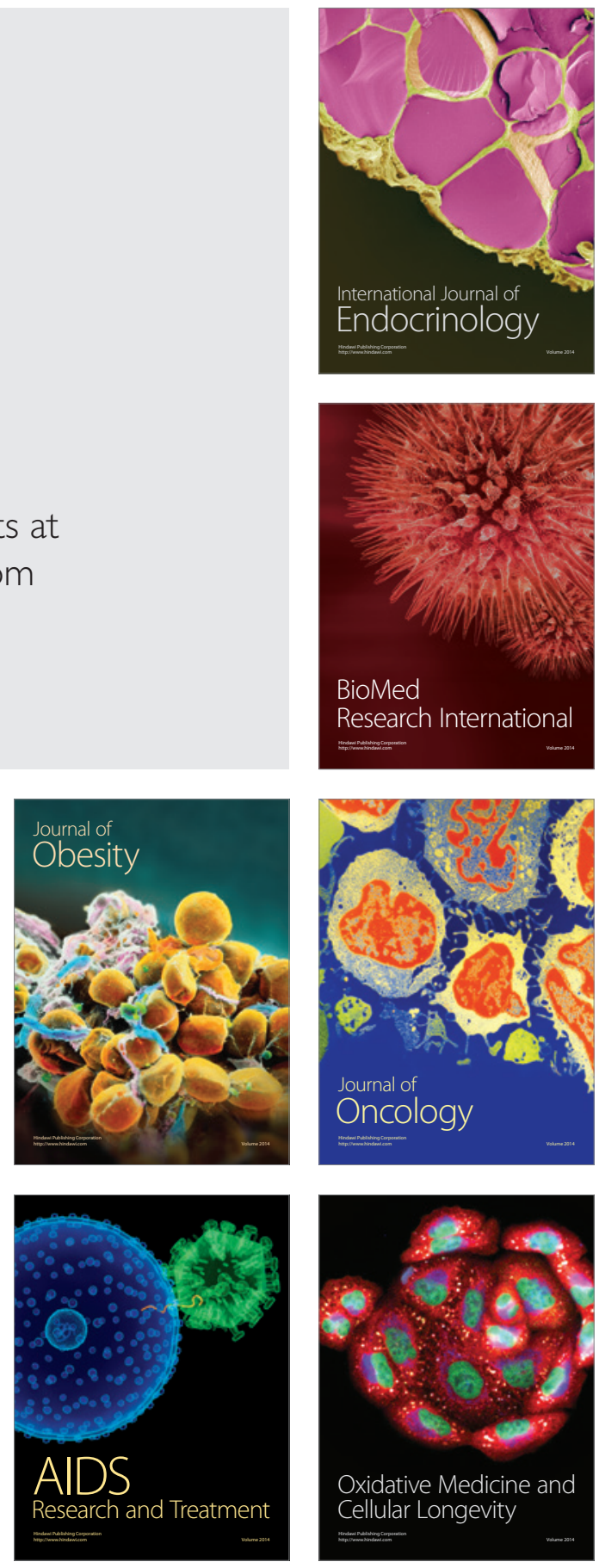